\section{TEM Specimen Preparation Technique For Small Semiconductor Devices}

\author{
Mark Hudson, John Benedict, and Philip Flaitz \\ IBM Microelectronics \\ hudsom@us.ibm.com
}

One of the methods of preparing samples for analysis by Transmission Electron Microscopy (TEM) is the well-known procedure using a Tripod polisher and the wedge technique (1-3), developed in our laboratory. Though developed explicitly for preparing samples of integrated circuit structures built on silicon, the technique has been used in our lab for a wide variety of other materials, including metals and ceramics. In general when working with silicon samples, we have the luxury of starting with large wafer pieces or chips that are generally at least a couple of millimeters square or larger. Recently, however, we needed to work for an extended period on GaAs based lasers, where the devices requiring analysis were individual lasers extracted from individual packages. These small samples, measuring $100 \times 100 \times 50$ microns, are too small for the manual handling involved in routine mechanical cross sectioning methods. A typical example of such a device can be seen in Figure 1 where the actual device, indicated by the arrow, has been detached from the package and is being held by the bond wire. Furthermore, we found the GaAs used in these lasers to be less robust mechanically than silicon during mechanical polishing. This article describes the method we developed for dealing with these tiny devices.

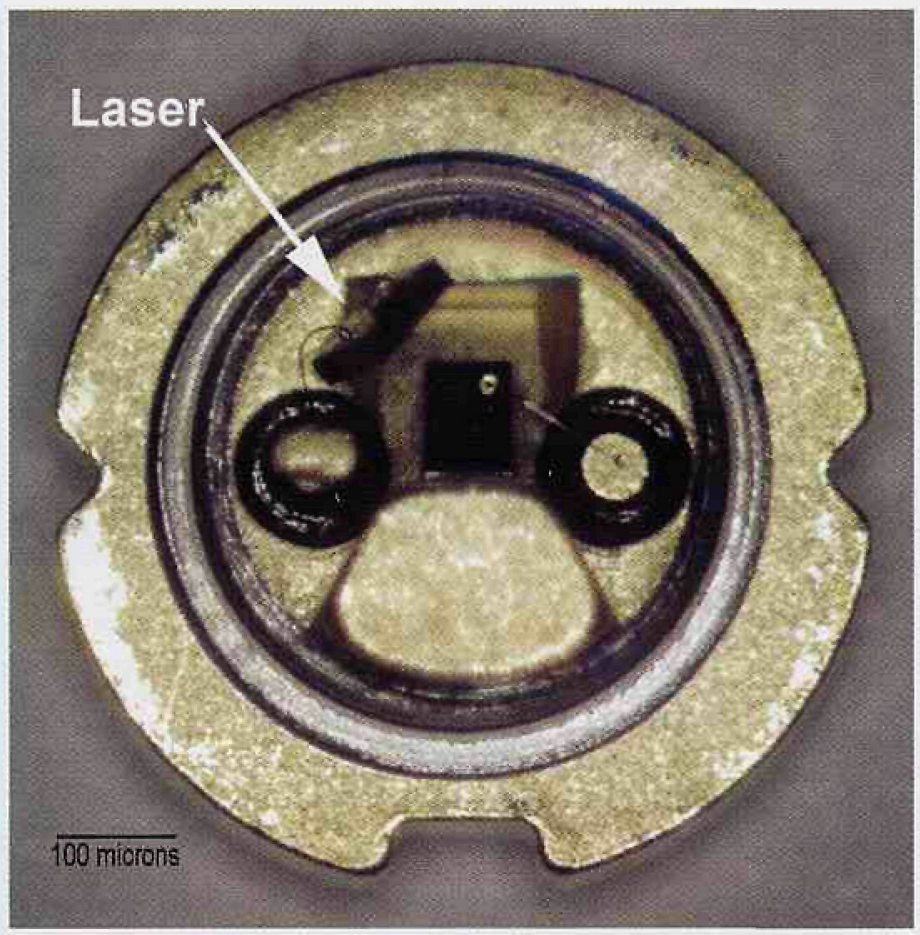

Figure 1 -- Depackaged laser device still attached to package by the bond wire.

A larger sample, for easier manual handling, is made by gluing the original small sample between a larger piece of silicon and a larger piece of dimpled quartz. The dimpled depression in the quartz is just large enough to surround the original sample. The sample is then mechanically thinned down using a Tripod polisher and the wedge technique. The quartz piece, glued to the top of the original sample, allows the progress of the polish to be monitored as the first side of the cross section is being mechanically polished. The silicon piece, glued to the bottom of the original sample, is used to gauge the final

\section{Device}

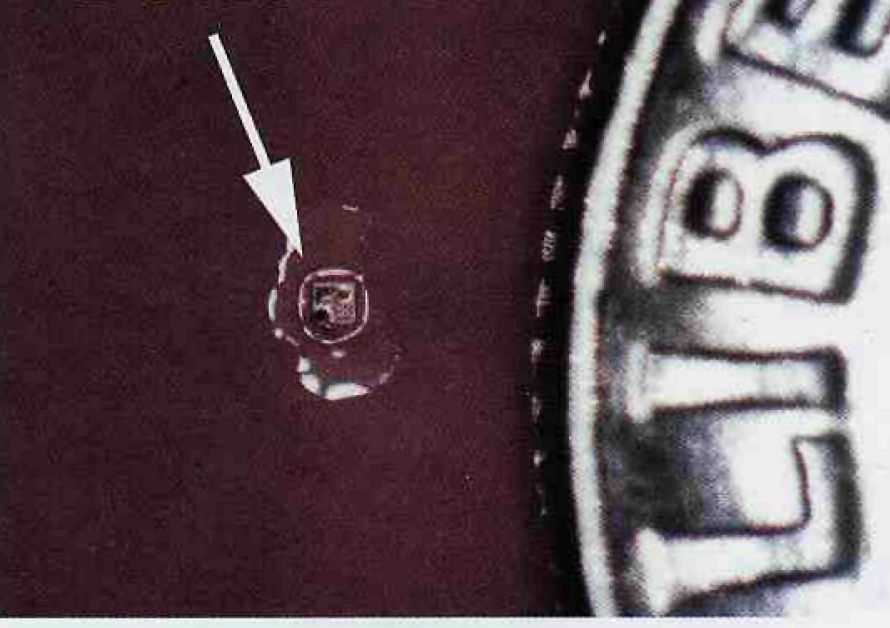

Figure 2 -- Laser device glued to silicon support piece next to a dime.

thickness of the wedge produced when polishing the second side of the cross section using the wedge technique. A final brief ion mill is used to remove any mechanical damage and to bring the device to electron transparency.

Before polishing, a pre-glue process is necessary where the three parts: the dimpled quartz piece, the silicon piece, and the sample are glued together in a stack. The first step is to mount the device to a piece of silicon, typically $10 \times 10 \mathrm{~mm}$ for easy handling. The gluing process starts with a very small drop of epoxy (we use Gatan G-1) that is applied to the rough, backside, of the silicon piece using the small tip of a toothpick. It is best to use a minimal amount of epoxy for this step, just enough to extend completely under the laser device. The laser is gently pressed down into the glue and the glue is allowed to cure at $80^{\circ} \mathrm{C}$ for 4 hours. The device, mounted on silicon, is seen in Figure 2, with a dime as a reference for size.

Next, a $10 \times 10 \times 1 \mathrm{~mm}$ square is cut from a piece of quartz. The quartz we used was a large block purchased from a local Science and Hobby store. The quartz block had to be cut down to a $10 \times 10 \mathrm{~mm}$ rod, then sliced into $1 \mathrm{~mm}$ thick pieces. This square is then mounted on the bottom of a Tripod polisher using glycol pthalate. The quartz is polished flat on both sides using diamond lapping film down to a $3 \mu \mathrm{m}$ finish, ending with a final thickness of $\sim 0.75 \mathrm{~mm}$. These polishing operations were required to remove the damage from cutting the quartz and make it possible to see through the quartz during the specimen preparation steps. After polishing, the quartz is taken off of the Tripod polisher and a dimple made in the center of the quartz piece using an ultrasonic cutting tool. We used a standard, solid, ultrasonic tip that we modified by fileing the tip down to a size slightly larger than the device we would

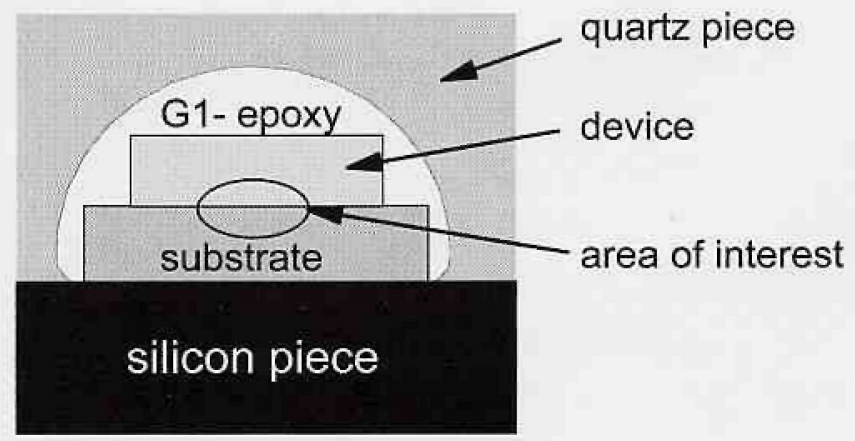

Figure 3 -- Diagram showing encapsulation structure. 


\section{EMC 2004}

\section{$13^{\text {th }}$ European Microscopy Congress}

\section{Plenary Lectures}

Wolfgang Baumeister: Biological tomography and $3 D$ reconstruction

Christian Colliex: Energy resolution, how low can we go?

Max Haider: HRTEM versus HRSTEM, competitive or complementary

\section{SPECIAL INTEREST LECTURES}

Bruno G. Brunetti: Microscopy in art \& archaeology

Ann De Veirman: Microscopy in (semiconductor) industry

Eduard Arzt: Microscopy in bio-nano technology

lan Parker: Microscopy with photons

Steven McDanels: Microscopic and metallurgical aspects of the Space Shuttle Columbia accident investigation and reconstruction

\section{SUNDAY COURSES (AUGuSt 22)}

Joachim Mayer: Interpretation of EELS spectra

Nigel Browning: STEM imaging

Richard Langford: FIB (focussed ion beam)

Hans Van der Voort: 3D reconstruction in biology

Niek F. van Hulst: Near field optical microscopy

\section{REGULAR SESSIONS (as currently planned)}

\section{Instrumentation and Methodology}

IM 1. Aberration correctors

IM 2. Lenses, filters, spectrometers, sources, detectors

IM 3. Quantitative HRTEM

IM 4. Quantitative diffraction, electron crystallography

IM 5. Nano-lab

IM 6. Electron holography

IM 7. Electron tomography

IM 8. EELS-EFTEM

IM 9. Low voltage SEM, variable pressure SEM IM 10. X-ray microscopy and microtomography IM 11. New microscopies and novelties

IM 12. Image analysis and processing

IM 13. Round table: so many exciting developments but what instrument to choose?

\section{Materials Sciences}

MS 1. Interface characterisation

MS 2. Nanoparticles and catalysts

MS 3. Nanostructured materials

MS 5. Magnetic materials

MS 6. Carbon and carbon based materials

MS 7. Si-based semiconductors

MS 8. Non Si-based semiconductors

MS 9. Ceramic materials

MS 10. Micro-meso-nano porous materials

MS 11. "Soft" materials and minerals

MS 12. Alloys and intermetallics
MS 13. Spectroscopy in materials science MS 14. FIB and advanced sample preparation MS 15. Surface structure

MS 16. Microscopy in art \& archaeology

\section{Life Sciences}

LS 1. Nucleus: structure and dynamics

LS 2. Immuno - in situ - reporter gene constructs

LS 3. Membrane trafficking

LS 4. The cytoskeleton

LS 5. Protein dynamics/interactins/FRAP-FRET

LS 6. Biomaterials

LS 7. Forensic microscopy

LS 8. LOW-voltage SEM

LS 9. Biological applications of scanning probe microscopy

LS 10. Cryo-electron microscopy/tomography of cells and organelles

LS 11. EM crystallography and quantitative electron diffraction

LS 12. Application of laser trapping and optical tweezers in biology

LS 13. Life cell imaging

LS 14. Single particle reconstruction of macromolecular aggregates

L5 15. Diagnostic EM in infectious diseases

LS 16. Sample preparation in life sciences

Extensive optional social programme,

including congress dinner, city tours, pre- \& post-congress tours,

Visit www.emc2004.be for the on-line registration procedure and latest updates or contact information@emc2004. be - tel. +32-3-265 3695 


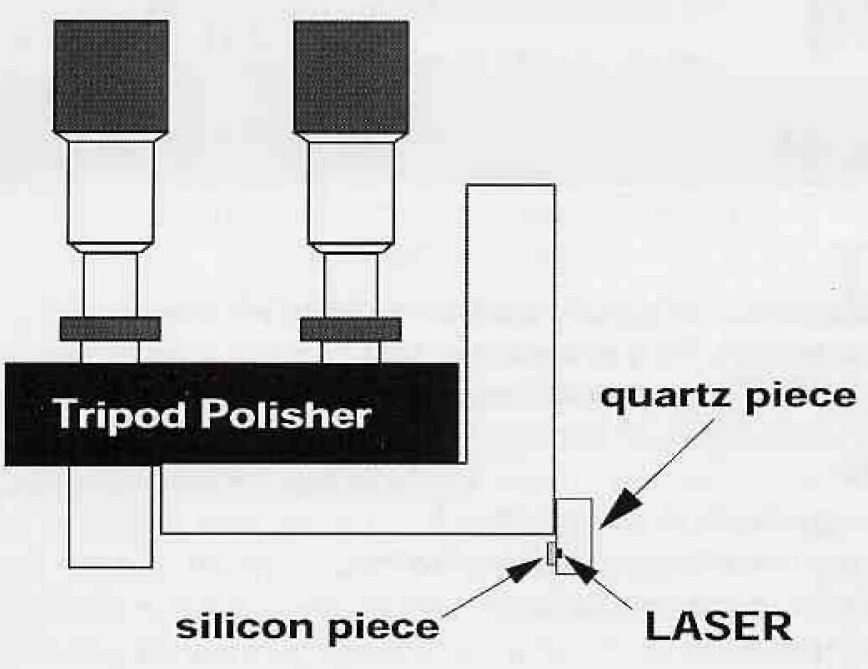

Figure 4 - Tripod mourting confrguration for first side polishing.

be mounting. The depth of the hole was gauged by placing the quartz over the mounted device, and cutting repeated until the quartz and silicon surfaces would meet without the quartz resting on the device. The quartz piece is now cleaned off in acetone and allowed to air dry.

After curing, the dimple in the quartz piece is filled with G-1 epoxy and a small drop of epoxy applied to the top of the device package with a toothpick. Filling the cavity and coating the device with fresh drops of epoxy minimizes the likelihood that an air bubble will be trapped around the device, causing difficulties during mechanical polishing. The dimple is then carefully inverted over the device package and the quartz is pressed down on to the silicon piece with the epoxy drops filling the cavity around the device and extruding out between the quartz and silicon, bonding the pieces together, as seen in Figure 3 . The dimple is then inspected to make sure that there are no air bubbles in the epoxy and the epoxy is allowed to cure overnight at $80^{\circ} \mathrm{C}$.

This silicon/device/quartz stack is now polished with a Tripod polisher. (South Bay Technology, Inc.) (4) Glycol phthalate is used to attach the stack to a Tripod polisher, with the quartz layer facing towards the front of the polisher Figure 4. The stack is then polished down to the device with progressively finer grits of diamond paper, using only 3 micron or finer to polish the device. The final polishing is done by using 0.25 micron diamond slurry on a "Final A" polishing cloth (Allied High Tech Products Inc.) for thirty seconds, followed by ten seconds of colloidal silica on the same cloth. Longer final polishing times cause rounding of the sample edges and should be avoided. The polished surface is then carefully wiped clean using soap and water, followed by acetone.

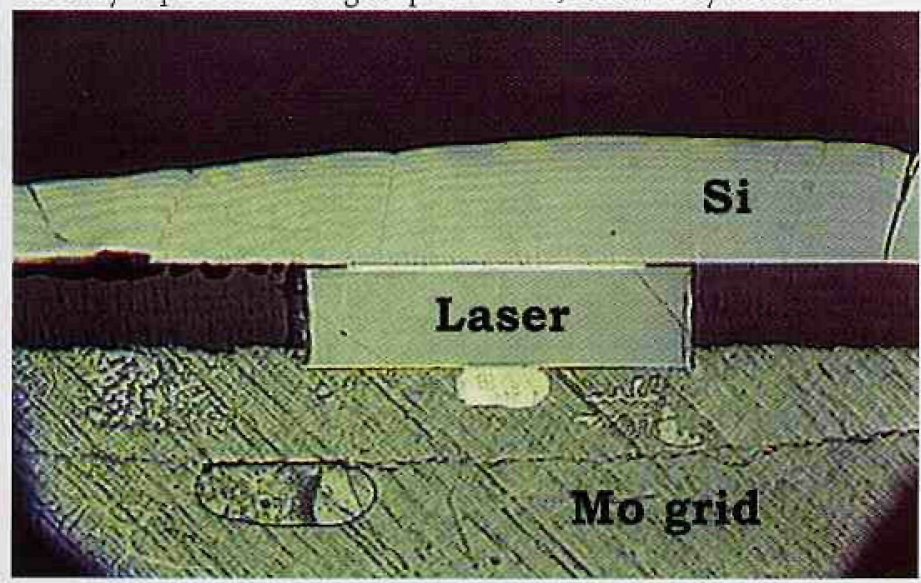

Figure 5 -- Optical image of final polished laser structure. Note the thickness fringes in the silicon support.

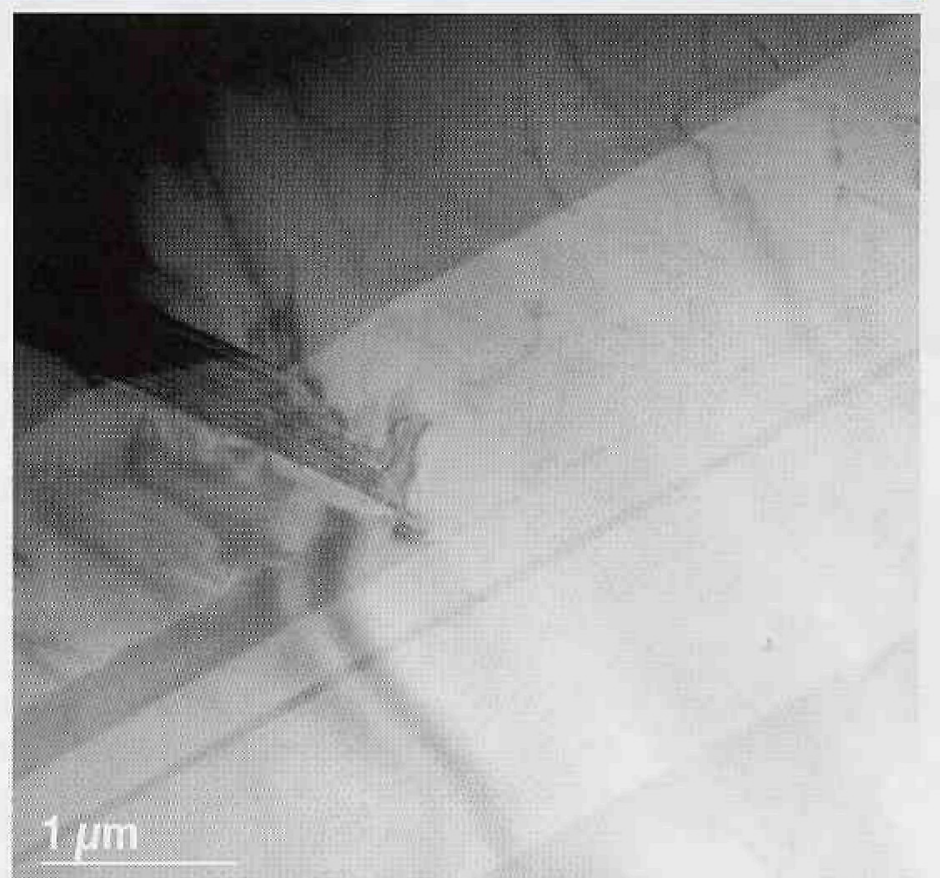

Figure 6 -- TEM image of a typical laser after a few minutes of Ar ion milling to remove polishing damage.

Before second side polishing, a grid is mounted to the first side polished surface. M-Bond 610 epoxy (Measurements Group Inc.) is painted on one side of a $0.4 \times 2 \mathrm{~mm}$ slotted molybdenum grid. The epoxy side of the grid is then carefully pressed down on the polished surface, aligning one edge of the grid with the back side of the laser device so that the laser/silicon interface is in the grid opening. The $\mathrm{M}$-Bond epoxy is now cured for two hours at $80^{\circ} \mathrm{C}$. The Tripod is prepared for wedge polishing using the method discussed in Benedict, et al. (1996). A small drop of cyanoacrylate (super) glue is placed in the slot of the grid and on the sample post of the Tripod polisher to minimize the incursion of air bubbles during mounting. The stack is oriented over the sample post so now the silicon layer is facing the front of the polisher. The grid is then carefully pressed down in the glue on the sample post and the excess glue around the grid is removed with a piece of filter paper. The cyanocrylate glue is cured overnight at $80^{\circ} \mathrm{C}$.

The rear feet of the Tripod are extended out an additional 100 microns to compensate for the thickness of the grid and provide sufficient wedge for the structural integrity of the sample. The stack is wet polished using diamond paper while monitoring the thickness of the wedge using the changes in transmitted light color of the silicon layer. The stack is mechanically polished until the transmitted light forms a multicolored fringe along the silicon-to-device interface. In cases where the laser substrate cannot be depackaged from the laser, the transmitted light fringes from that part of the device can be used to gauge the final thickness. This can be seen in Figure 5, with the various parts of the specimen identified. The device is thinned to TEM transparency using low angle Ar ion milling and monitoring the thinning process with the TEM. A typical example of a final thinned laser is seen in Figure 6.

Using this technique, we have successfully prepared a large number of laser devices, and have adapted the technique to other structures too small to be prepared by Tripod polishing directly.

\section{References}

1. S.J. Klepeis et al., Mater. Res. Soc. Proc. 115 (1988) 179

2. J.P. Benedict et al., Mater. Res. Soc. Proc. 199 (1990) 189

3. J.P. Benedict et al,, Microstructure Science, ASM International 23 (1996) 277.

4. The Tripod Polisher as well as glycol phthalate wax and other supplies is available from South Bay Technology, Inc. (www.southbaytech.com). 


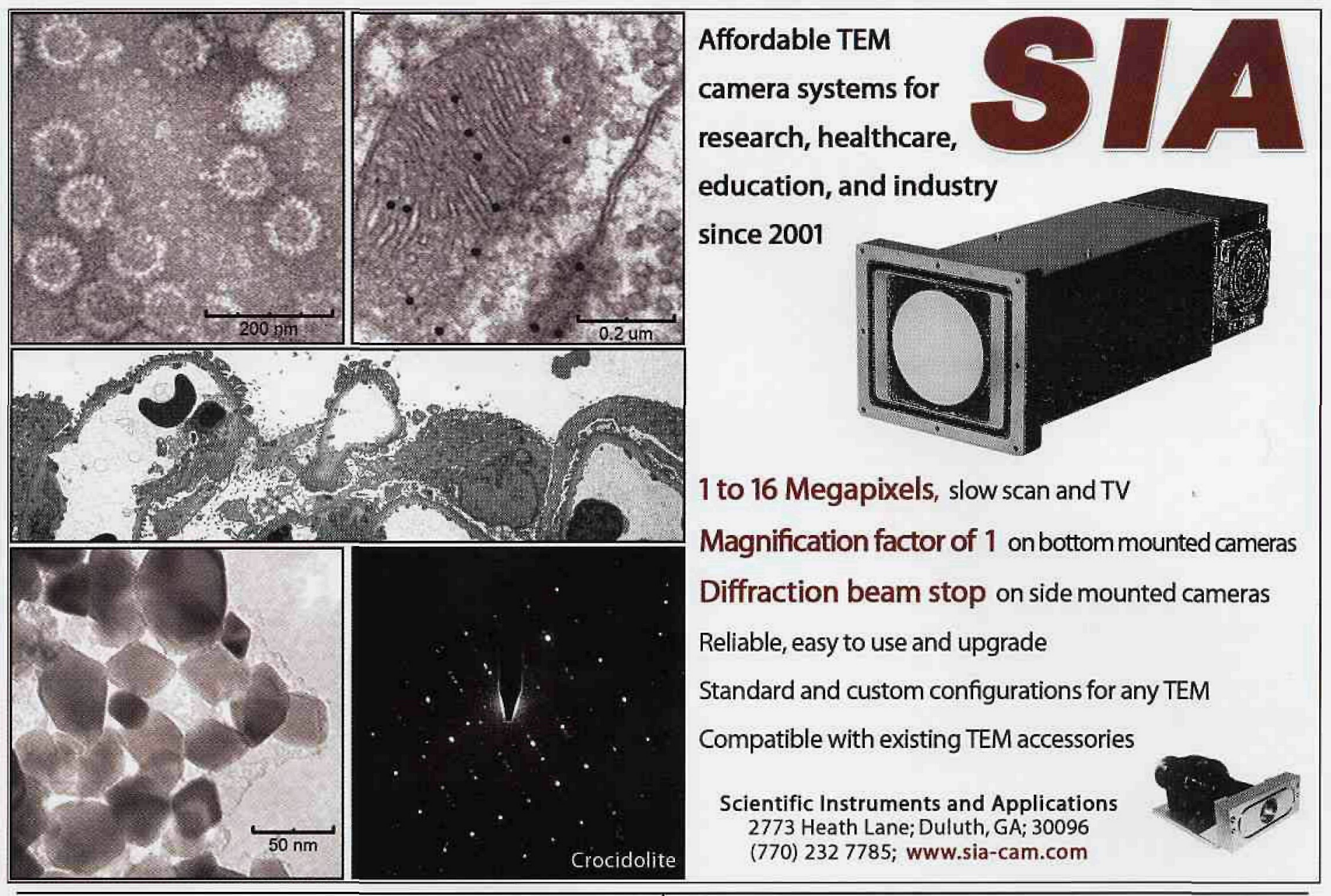

\section{Nifreserpy Auetions}

- Over 200 Microscope ads online daily

- Buy \& Sell all major brands

- Free "Wanted" ads for quick resultis

Naw - Eurplug - USEd Microscopes, Accessories, Objectives 